\title{
Association of NEFA composition with insulin sensitivity and beta cell function in the Prospective Metabolism and Islet Cell Evaluation (PROMISE) cohort
}

\author{
Luke W. Johnston $^{1}$ • Stewart B. Harris ${ }^{2}$ • Ravi Retnakaran ${ }^{3,4}$ - Adria Giacca ${ }^{5}$ - Zhen Liu ${ }^{1}$ - Richard P. Bazinet ${ }^{1}$. \\ Anthony J. Hanley ${ }^{1,3,6}$
}

Received: 8 August 2017 / Accepted: 14 November 2017 / Published online: 24 December 2017

(C) Springer-Verlag GmbH Germany, part of Springer Nature 2017

\begin{abstract}
Aims/hypothesis Our aim was to determine the longitudinal associations of individual NEFA with the pathogenesis of diabetes, specifically with differences in insulin sensitivity and beta cell function over 6 years in a cohort of individuals who are at risk for diabetes.

Methods In the Prospective Metabolism and Islet Cell Evaluation (PROMISE) longitudinal cohort, 477 participants had serum NEFA measured at the baseline visit and completed an OGTT at three time points over 6 years. Outcome variables were calculated using the OGTT values. At each visit, insulin sensitivity was assessed using the HOMA2 of insulin sensitivity (HOMA2-\%S) and the Matsuda index, while beta cell function was assessed using the insulinogenic index over HOMA-IR (IGI/IR) and the insulin secretion-sensitivity index-2 (ISSI-2). Generalised estimating equations were used, adjusting for time, waist, sex, ethnicity, baseline age, alanine aminotransferase (ALT) and physical activity. NEFA were analysed as both concentrations $(\mathrm{nmol} / \mathrm{ml})$ and proportions $(\mathrm{mol} \%)$ of the total fraction.

Results Participants' (73\% female, 70\% with European ancestry) insulin sensitivity and beta cell function declined by $14-21 \%$ over 6 years of follow-up. In unadjusted models, several NEFA (e.g. 18:1 n-7, 22:4n-6) were associated with lower insulin sensitivity, however, nearly all of these associations were attenuated in fully adjusted models. In adjusted models, total NEFA, 16:0, 18:1 n-9 and 18:2 n-6 (as concentrations) were associated with 3.7-8.0\% lower IGI/IR and ISSI-2, while only 20:5 n-3 (as mol\%) was associated with $7.7 \%$ higher HOMA2-\%S.

Conclusions/interpretation Total NEFA concentration was a strong predictor of lower beta cell function over 6 years. Our results suggest that the association with beta cell function is due to the absolute size of the serum NEFA fraction, rather than the specific fatty acid composition.
\end{abstract}

Keywords Beta cell function · Diabetes pathogenesis · Fatty acid composition · Insulin sensitivity · Longitudinal cohort . Non-esterified fatty acids

Electronic supplementary material The online version of this article (https://doi.org/10.1007/s00125-017-4534-6) contains peer-reviewed but unedited supplementary material, which is available to authorised users.

Anthony J. Hanley

anthony.hanley@utoronto.ca

1 Department of Nutritional Sciences, Faculty of Medicine, University of Toronto, FitzGerald Building, 150 College Street,

Toronto, ON M5S 3E2, Canada

2 Centre for Studies in Family Medicine, University of Western Ontario, London, ON, Canada
3 Division of Endocrinology, University of Toronto, Toronto, ON, Canada

4 Lunenfeld Tanenbaum Research Institute, Mount Sinai Hospital, Toronto, ON, Canada

5 Department of Physiology, University of Toronto, Toronto, ON, Canada

6 Dalla Lana School of Public Health, University of Toronto, Toronto, ON, Canada 


\section{Research in context}

\section{What is already known about this subject?}

- Serum fatty acids play an important role in glucose regulation and metabolism and, as such, influence the pathophysiology of type 2 diabetes

- Fatty acids are a diverse class of molecule with a broad range of physiological functions, and this diversity is reflected in the serum composition

- Higher total serum NEFA is an established risk factor for type 2 diabetes

\section{What is the key question?}

- What is the influence of the specific NEFA composition on insulin sensitivity and beta cell function over time?

\section{What are the new findings?}

- There were no associations of the specific NEFA composition on insulin sensitivity and beta cell function

- Higher total serum NEFA was strongly associated with lower beta cell function over 6 years of follow-up

\section{How might this impact on clinical practice in the foreseeable future?}

- These results reinforce the view that quantifying total NEFA remains a useful clinical measure and that measuring the composition does not contribute additional information

$\begin{array}{ll}\text { Abbreviations } & \\ \text { ALT } & \text { Alanine aminotransferase } \\ \text { EPA } & \text { Eicosapentaenoic acid } \\ \text { FDR } & \text { False discovery rate } \\ \text { GEE } & \text { Generalised estimating equations } \\ \text { HOMA2-\%S } & \text { HOMA2 of insulin sensitivity } \\ \text { IFG } & \text { Impaired fasting glucose } \\ \text { IGI/IR } & \text { Insulinogenic index over HOMA-IR } \\ \text { IGT } & \text { Impaired glucose tolerance } \\ \text { ISI } & \text { Insulin sensitivity index (Matsuda index) } \\ \text { ISSI-2 } & \text { Insulin secretion-sensitivity index-2 } \\ \text { MAQ } & \text { Modified activity questionnaire } \\ \text { MET } & \text { Metabolic equivalent of task } \\ \text { PLS } & \text { Partial least squares regression } \\ \text { PROMISE } & \text { Prospective Metabolism and Islet Cell } \\ & \text { Evaluation Cohort } \\ \text { PUFA } & \text { Polyunsaturated fatty acids } \\ \text { QIC } & \text { Quasi-likelihood information criterion } \\ \text { TAG } & \text { Triacylglycerol } \\ \text { WC } & \text { Waist circumference }\end{array}$

\section{Introduction}

Total NEFA has been well documented to influence the pathogenesis of type 2 diabetes mellitus. Experimental work has shown that exposure to high concentrations of NEFA can induce insulin resistance in insulin-sensitive tissues such as muscle and liver, and can impair pancreatic beta cell production of insulin $[1,2]$. Observational and clinical studies have reported concordant findings, showing in particular, that elevated total plasma NEFA associates with an increased risk for incident type 2 diabetes [3, 4].

Much of the previous experimental work on the role of NEFA in type 2 diabetes utilised individual fatty acids such as palmitic acid (16:0) or oleic acid (18:1n-9) or alternatively used specific oils such as soybean oil, which is high in the polyunsaturated fatty acid (PUFA) linoleic acid (18:2 n-6), as the exposure to characterise the metabolic impact of total NEFA. However, fatty acids comprise multiple molecules with diverse physiological functions and few studies have analysed the effects of a broader spectrum of fatty acids. For instance, one study suggested that eicosapentaenoic acid (EPA; 20:5 n-3) can protect against the lipotoxic effect of palmitic acid in the beta cells [5].

Despite a sizeable literature investigating the role of total NEFA concentration in diabetes, there are important gaps in this research. The majority of previous studies have used animal models or cell lines [6, 7], have been short term human trials [8-11] or have been epidemiological studies that only looked at total NEFA and not individual fatty acids [12-14]. To date, there have been no longitudinal studies examining the role of the serum NEFA composition on the pathogenesis of diabetes, which is critical given the protracted natural history of diabetes and the growing appreciation of the divergent effects of individual fatty acids. Therefore, our objective was to examine the association between serum NEFA composition with differences over time in insulin sensitivity and beta cell function in a longitudinal cohort. We hypothesised that higher palmitic acid and lower PUFA, such as EPA, in the 
NEFA fraction would associate with declining insulin sensitivity and beta cell function over 6 years.

\section{Methods}

Participants from London and Toronto, Canada, were recruited into the Prospective Metabolism and Islet Cell Evaluation (PROMISE) cohort. Eligibility for recruitment into PROMISE required having one or more risk factors for type 2 diabetes mellitus including obesity, hypertension, family history of diabetes and/or a history of gestational diabetes or giving birth to a macrosomic infant. Participants aged 30 years and older $(n=736)$ attended the baseline visit between 2004 and 2006. Follow-up examinations in this cohort occurred every 3 years, with three examination visits completed to date (2004-2006, 2007-2009 and 2010-2013). Participants were contacted annually by telephone. The current study used data on participants who did not have diabetes at baseline, who returned for one or more of the follow-up examinations and had samples available for fatty acid measurements $(n=477)$. A diagram showing the sample size at each visit is shown in ESM Fig. 1. At each examination, participants underwent metabolic characterisation, anthropometric measurements and questionnaires on lifestyle and sociodemographic factors. Research ethics approval was obtained from Mount Sinai Hospital and the University of Western Ontario and all participants provided written informed consent. Data collection methods were standardised across the two centres and research nurses were centrally trained.

Blood measurements At each examination, an 8-12 h fasting blood sample was drawn from each participant, followed by a $75 \mathrm{~g}$ OGTT with a blood draw at $30 \mathrm{~min}$ and $2 \mathrm{~h}$. All blood samples were processed and frozen at $-70^{\circ} \mathrm{C}$. Alanine aminotransferase (ALT) was measured using standard laboratory procedures. Cholesterol, HDL and triacylglyceride (TAG) were measured using Roche Modular's enzymatic colorimetric tests (Roche Canada, Mississauga, ON, Canada). Both insulin and glucose were derived from the OGTT at fasting, $30 \mathrm{~min}$ and $2 \mathrm{~h}$. Specific insulin was measured using the Elecsys 1010 (Roche Diagnostics, Basel, Switzerland) immunoassay analyser and electrochemiluminescence immunoassay. This assay shows $0.05 \%$ cross-reactivity to intact human proinsulin and the Des 31,32 circulating split form with a CV of $9.3 \%$. Glucose was determined using an enzymatic hexokinase assay (Roche Canada) with a detection range of 0.11 to $41.6 \mathrm{mmol} / \mathrm{l}$. The inter-assay CV is $<1.1 \%$ and intra-assay CV is $<1.9 \%$. All assays were performed at the Banting and Best Diabetes Centre Core Lab at Mt. Sinai Hospital. Impaired fasting glucose (IFG), impaired glucose tolerance (IGT) and diabetes were categorised using the 2006 WHO criteria [15].
NEFA composition was quantified using stored fasting serum samples from the baseline visit, which had been frozen at $-70^{\circ} \mathrm{C}$ for $4-6$ years and had not been exposed to any freezethaw cycles. Serum fatty acids have been documented to be stable at these temperatures for up to 10 years [16]. A known amount of heptadecanoic acid (17:0) was added to the serum as an internal standard prior to extracting total lipids according to the method of Folch et al 1957 [17]. Each serum lipid fraction (NEFA, cholesteryl ester, phospholipid and TAG) was isolated using thin layer chromatography; each fraction was visualised under UV light after lightly spraying with 8anilino-1-naphthalene sulfonic acid $(0.1 \% \mathrm{wt} / \mathrm{vol}$.) and then converted to fatty acid methyl esters with $14 \%$ boron trifluoride in methanol at $100^{\circ} \mathrm{C}$ for $1 \mathrm{~h}$. Fatty acid methyl esters were separated and quantified using a Varian-430 gas chromatograph (Varian, Lake Forest, CA, USA) equipped with a Varian Factor Four capillary column and a flame ionisation detector, which were injected in splitless mode. Fatty acid concentrations $(\mathrm{nmol} / \mathrm{ml})$ were calculated by proportional comparison of gas chromatography peak areas to that of the internal standards [18]. There were 22 fatty acids measured in the NEFA fraction. Given their diverse biology, as well as the complexity of the analyses, findings for other lipid fractions in this cohort are reported separately (see Johnston et al 2016 [19] for the analysis of the phospholipid and cholesteryl ester fractions).

Outcome variables Insulin sensitivity and beta cell function indices were computed using the OGTT glucose and insulin data. Insulin sensitivity was assessed using the HOMA2 of insulin sensitivity (HOMA2-\%S) [20] and the insulin sensitivity index (ISI) [21]. HOMA2-\%S largely reflects hepatic insulin sensitivity, while ISI reflects whole-body insulin sensitivity [22]. Beta cell function was assessed using the insulinogenic index [23] over HOMA-IR (IGI/IR) and the insulin secretion-sensitivity index-2 (ISSI-2) [24]. IGI/IR is a measure of the first phase of insulin secretion while ISSI-2 is analogous to the disposition index (but using OGTT values). Each index has been validated against gold standard measures $[21,24,25]$. Formulas used to calculate these measures can be found in the ESM Methods: Equations for outcomes.

Anthropometrics and sociodemographic factors Height, weight and waist circumference (WC) were measured at all clinic examinations using standard procedures. WC was measured at the natural waist, defined as the narrowest part of the torso between the umbilicus and the xiphoid process. BMI was calculated by dividing weight $(\mathrm{kg})$ by height $(\mathrm{m})$ squared. Sociodemographic information, including age, sex and ethnicity, were determined using questionnaires administered at each examination. In the lifestyle questionnaire, physical activity was determined using a version of the modifiable activity questionnaire (MAQ) [26]. The MAQ collects information 
on leisure and occupational activity, including intensity, frequency and duration, over the past year. Each reported activity from the MAQ was weighted by its metabolic intensity allowing for the estimation of metabolic equivalent of task (MET) hours per week (MET-h/week).

Statistical analysis The primary outcome variables for this analysis were HOMA2-\%S, ISI, IGI/IR and ISSI-2; outcome variables were log-transformed for the statistical modelling. The primary predictor variables for this analysis were 22 individual NEFA as molar percentages ( $\mathrm{mol} \%$ ) of the total fraction and as concentrations (nmol/ml). Pearson correlation coefficients were computed to assess the relationships of individual NEFA with other continuous variables.

For the primary analysis, generalised estimating equation (GEE) models [27] were used to determine the longitudinal associations between the outcome variables and the predictor variables. Given the longitudinal design an auto-regressive of order 1 correlation matrix was chosen for the GEE models, though other matrices (e.g. exchangeable) had similar fit (data not shown). GEE is well suited to longitudinal cohort studies given its capacity to handle any missed visits and for the correlation of variables between time points. See ESM Methods: Further description of statistical methods for more detail on GEE. The predictor variables and continuous covariates were scaled (mean centred and standardised). The NEFA variables were classified as time-independent (held constant) as they were measured only at the baseline visit, while the outcome variables were set as time-dependent. No imputation was conducted on missing values and incident diabetes cases were excluded from the analysis (follow-up data were included until date of diagnosis). A GEE model is applied for each combination of outcome and NEFA (as either mol\% or $\mathrm{nmol} / \mathrm{ml})$.

Covariate selection was based on previous literature, directed acyclic graph [28-30] recommendations (see ESM Fig. 2 and ESM Fig. 3) and quasi-likelihood information criterion (QIC) [31]. See ESM Methods: Further description of statistical methods for more detail on QIC and the directed acyclic graphs. ESM Table 1 shows the covariates compared using QIC. We selected the model (M9) that had the fewest covariates and that had similar fit between the outcome measures. The final GEE model included the covariates years from baseline (time), sex (female or male), ethnicity (European ancestry or non-European ancestry), baseline age, WC, ALT and MET-h/week (physical activity). Sex, ethnicity and baseline age were time-independent variables. See ESM Methods: Further description of statistical methods for more detail on the covariate inclusion. After scaling and log-transforming the model variables, then exponentiating the GEE estimates, the interpretation of the GEE results is as an expected percentage difference in the outcome variable for every SD increase in the predictor variable, for the same covariate values (e.g. same age or sex). With the main effect associations (not with a time interaction), this interpretation is applied consistently at each time point and thus represents associations of baseline NEFA for each future value of the outcomes. Since TAG is a risk factor for diabetes and since NEFA contribute to TAG production [32], TAG may act as a mediator between NEFA and the outcomes. To determine the role of TAG in the association between NEFA and the outcomes, TAG was included in the GEE model in a sensitivity analysis. Finally, for the GEE models, we tested for an interaction with sex, ethnicity or time by the predictor term for each outcome variable.

While GEE accounts for the longitudinal design of the data, this approach is limited in that it cannot analyse the inherent multivariate nature of the composition of the NEFA fraction, as each model only contains data from a single fatty acid. Therefore, to confirm the results of the GEE analyses in a multivariate environment (with all NEFA variables in a single model), partial least squares (PLS) was used with the outcomes as the dependent variable.

All analyses were performed using R 3.4.2 [33], along with the R packages geepack 1.2.1 for GEE [34] and the package pls 2.6.0 for the PLS analysis. The R code and output for all analyses of this manuscript is available at https://oi.org/10. 6084/m9.figshare.3472433. Results were considered statistically significant at $p<0.05$, after adjusting for multiple testing using the Benjamini-Hochberg false discovery rate (FDR) [35]. Strengthening the Reporting of Observational Studies in Epidemiology (STROBE) was used as a guideline for reporting [36].

\section{Results}

Basic characteristics of the PROMISE cohort For this study, there were $349(73.2 \%)$ females and $336(70.4 \%)$ had European ancestry (see Table 1). The mean follow-up time was $5.6 \pm 1.0$ years, where $88.7 \%$ of participants attended all three visits. The mean age of the participants at baseline was $50.1 \pm 9.8$ years and the mean BMI was $31.1 \pm 6.4 \mathrm{SD} \mathrm{kg} / \mathrm{m}^{2}$. Most of the participants $(64.8 \%)$ had a family history of diabetes. Insulin sensitivity and beta cell function measures showed a significant median decline from baseline to 6 years of between $14 \%$ to $21 \%$ ( $p<0.001$, based on a time-adjusted GEE model) in this analysis. Consistent with this decline, there were $42(9 \%)$ and $96(20 \%)$ individuals diagnosed with diabetes and pre-diabetes (IFG and IGT), respectively, over the 6 years. Individuals with diabetes were excluded from GEE analyses (1, 28 and 13 individuals were excluded at 0 , 3 and 6 year visits, respectively). Follow-up data were included until date of diagnosis. Certain medications (e.g. lipid medications such as statins or fibrates) could influence the association between NEFA and the outcomes. There were 60 and 103 participants who used lipid modifying medications at the 
Table 1 Basic characteristics of the PROMISE participants at each of the three clinic visits

\begin{tabular}{|c|c|c|c|}
\hline Measure & Baseline & 3 years & 6 years \\
\hline Sample size $(n)$ & 477 & 476 & 423 \\
\hline HOMA2-\%S & $88.8(54.2-136.7)$ & $76.8(49.1-121.8)$ & $73.7(49.5-110.1)$ \\
\hline ISI & $13.6(8.7-21.8)$ & $11.6(6.9-19.1)$ & $11.6(7.5-17.5)$ \\
\hline IGI/IR & $7.1(4.2-10.6)$ & $5.6(3.6-9.8)$ & $5.6(3.5-9.0)$ \\
\hline ISSI-2 & $727.5(570.0-922.5)$ & $613.4(493.9-836.7)$ & $622.5(472.5-810.3)$ \\
\hline BMI $\left(\mathrm{kg} / \mathrm{m}^{2}\right)$ & $31.1 \pm 6.4$ & $31.4 \pm 6.5$ & $31.1 \pm 6.6$ \\
\hline $\mathrm{WC}(\mathrm{cm})$ & $98.5 \pm 15.5$ & $99.3 \pm 15.7$ & $100.4 \pm 15.7$ \\
\hline Age (years) & $50.1 \pm 9.8$ & $53.2 \pm 9.7$ & $56.5 \pm 9.6$ \\
\hline MET-h/week & $45.2 \pm 59.7$ & $48.5 \pm 60.5$ & $44.1 \pm 57.1$ \\
\hline $\operatorname{ALT}(\mathrm{U} / \mathrm{l})$ & $29.6 \pm 16.0$ & $28.4 \pm 19.5$ & $25.9 \pm 16.9$ \\
\hline TAG $(\mathrm{mmol} / \mathrm{l})$ & $1.5 \pm 0.8$ & $1.4 \pm 0.8$ & $1.4 \pm 0.7$ \\
\hline Cholesterol (mmol/l) & $5.2 \pm 0.9$ & $5.1 \pm 1.0$ & $5.1 \pm 0.9$ \\
\hline HDL (mmol/l) & $1.4 \pm 0.4$ & $1.3 \pm 0.4$ & $1.4 \pm 0.4$ \\
\hline NEFA (nmol/ml) & $383.1 \pm 116.3$ & & \\
\hline Fasting glucose (mmol/l) & $5.0 \pm 0.5$ & $5.3 \pm 0.8$ & $5.3 \pm 0.7$ \\
\hline $2 \mathrm{~h}$ glucose $(\mathrm{mmol} / \mathrm{l})$ & $5.7 \pm 1.4$ & $6.5 \pm 2.3$ & $6.4 \pm 1.8$ \\
\hline Fasting insulin (pmol/l) & $75.3 \pm 56.3$ & $83.8 \pm 64.1$ & $85.9 \pm 57.0$ \\
\hline $2 \mathrm{~h}$ insulin $(\mathrm{pmol} / \mathrm{l})$ & $428.0 \pm 368.4$ & $528.1 \pm 496.6$ & $480.4 \pm 416.6$ \\
\hline \multicolumn{4}{|l|}{ Ethnicity } \\
\hline European & $336(70)$ & & \\
\hline Latino & $58(12)$ & & \\
\hline Other & $51(11)$ & & \\
\hline South Asian & $32(7)$ & & \\
\hline \multicolumn{4}{|l|}{ Sex } \\
\hline Female & 349 (73) & & \\
\hline Male & $128(27)$ & & \\
\hline
\end{tabular}

Values are in median (IQR), mean $\pm \mathrm{SD}$, or $n(\%)$

The proportion of ethnic and sex groups did not change over the 6 years

Outcome variables sample size ranges from $367-470$ over the 3 visits baseline and 6 year visit, respectively. Use of these medications did not influence NEFA composition, the outcomes or their associations as analysed from the GEE models (data not shown).

Figure 1 shows the compositional distribution of NEFA in the study participants. Four individual NEFA made up the vast majority $(89.3 \%)$ of the total NEFA fraction. The largest contributors were 18:1 n-9 (36.6\%), 16:0 (23.4\%), 18:0 (15.2\%) and 18:2 $n-6(14.1 \%)$. Raw concentration values are shown in ESM Table 2. All individual NEFA as well as the total fraction had correlations that ranged from weak to null $(r<0.3)$ with participant characteristics (see ESM Fig. 4 and ESM Fig. 5).

GEE modelling A number of associations were seen in the unadjusted GEE models (see Fig. 2), particularly for NEFA modelled as a concentration. There were several fatty acids (18:1 n-7, 20:5 n-3, 22:1 n-9, 22:4 n-6, 20:4 n-6, 16:0, 18:1 n$9,20: 2 n-6$ and total NEFA) that had significant associations with both insulin sensitivity and beta cell function outcomes.

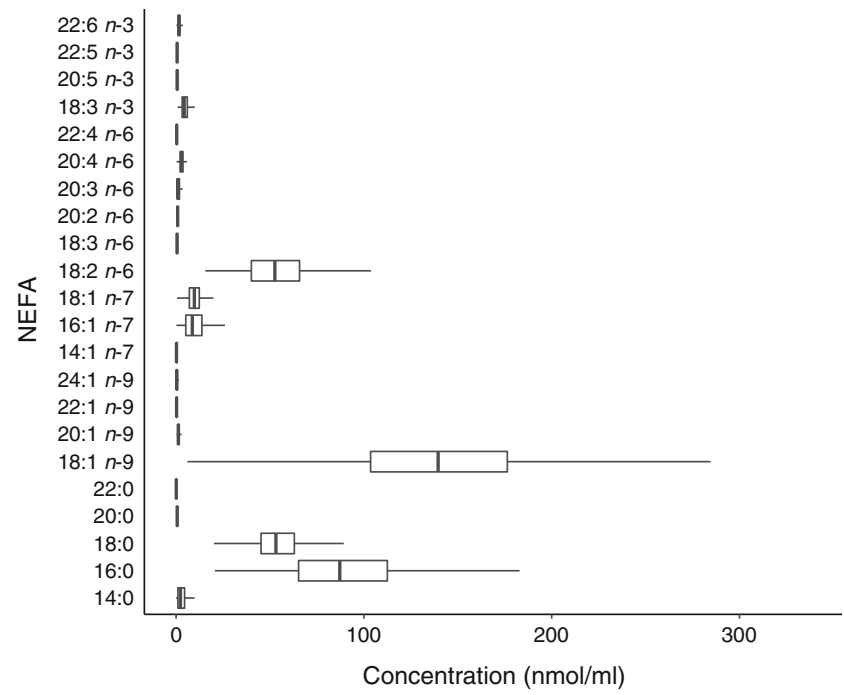

Fig. 1 Concentrations $(\mathrm{nmol} / \mathrm{ml})$ of each NEFA in PROMISE participants at the baseline visit (2004-2006) 
Fig. 2 Longitudinal associations of individual NEFA (mol\% and $\mathrm{nmol} / \mathrm{ml}$ ) with insulin sensitivity and beta cell function over 6 years in the PROMISE cohort. Models are only adjusted for time. $x$-axis values represent a percentage difference with $95 \% \mathrm{CI}$ in the outcome per SD increase in the fatty acid. $p$ values were adjusted for the Benjamini-Hochberg FDR. The larger black dot represents a statistically significant association at $p<0.05$
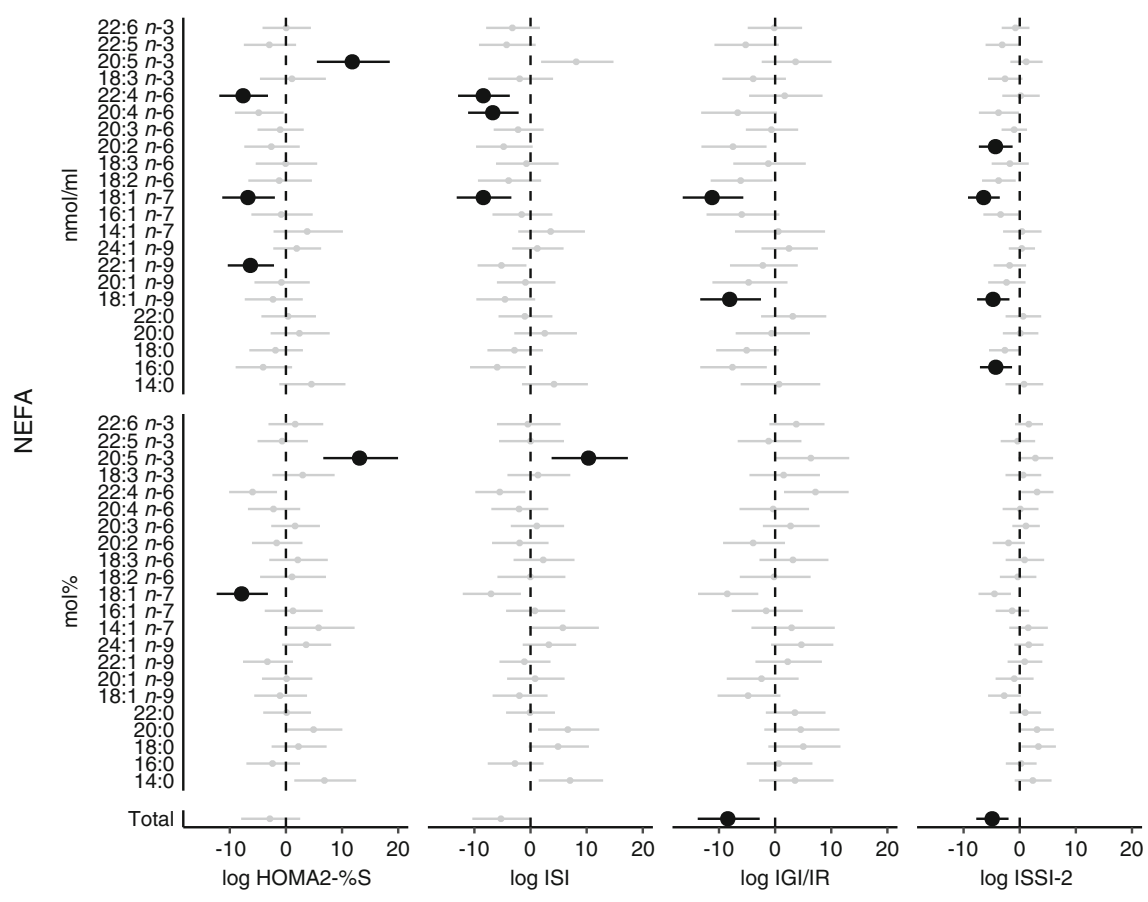

Per cent difference $(95 \% \mathrm{Cl})$ in the outcomes for each SD increase in NEFA
Most associations were negative, however, only 20:5 n-3 (EPA) had positive associations with insulin sensitivity as both a mol\% and as a concentration. One fatty acid, 18:1 n-7 (vaccenic acid), had consistent negative associations with all four outcomes.
After full model adjustment (Fig. 3), most significant associations were attenuated, particularly with insulin sensitivity. The remaining fatty acids (20:5 n-3 [EPA], 16:0, 18:1 n-9, 18:2 $n-6$ and total NEFA) had significant associations, of which only EPA had a significant association (with
Fig. 3 Longitudinal associations of individual NEFA (mol\% and $\mathrm{nmol} / \mathrm{ml}$ ) with insulin sensitivity and beta cell function over the 6 years in the PROMISE cohort. GEE models were fully adjusted. $x$-axis values represent a percentage difference with $95 \%$ $\mathrm{CI}$ in the outcome per SD increase in the fatty acid. $p$ values were adjusted for the BenjaminiHochberg FDR. The larger black dot represents a statistically significant association at $p<0.05$
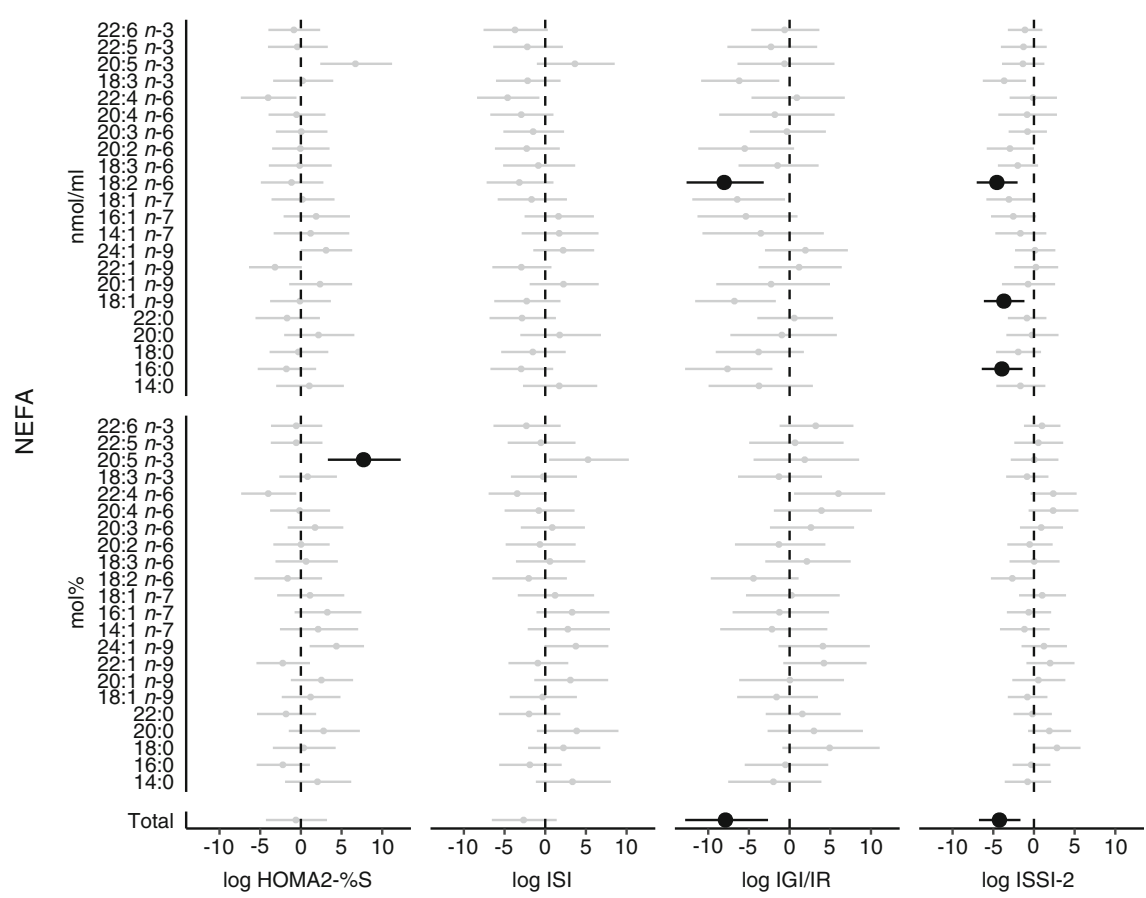

Per cent difference $(95 \% \mathrm{Cl})$ in the outcomes for each SD increase in NEFA 
HOMA2-\%S) as a mol\% and was the only fatty acid with a positive association. All other fatty acids had negative associations with IGI/IR and ISSI-2. The magnitude of association for each of these variables was fairly consistent for each beta cell function measure. For every one SD increase in any of the NEFA variables associated with beta cell function, there was an average predicted $7.7 \%$ lower IGI/IR and $8 \%$ lower ISSI2 at any given clinic visit.

Sensitivity analyses revealed that adjustment for WC was responsible for attenuating the unadjusted results (see ESM Fig. 6). Adjusting for TAG attenuated all associations with IGI/IR and ISSI-2 (data not shown), though this may be due to the fact that only a subset of participants at the 6 year visit had TAG measured. Sensitivity analyses examining associations with the sum of fatty acid classes (saturated vs unsaturated) revealed similar findings as compared with the adjusted GEE model for total NEFA (data not shown). There were no significant interaction effects of time, sex or ethnicity in either unadjusted or adjusted models. Medication use for controlling lipid and cholesterol did not influence the results (data not shown). Raw values from the GEE models are shown in ESM Table 3 for unadjusted models and ESM Table 4 for fully adjusted models. Consistent with the GEE modelling, results from modelling all NEFA in a multivariate environment using PLS showed no discernible clustering nor was it predictive of the outcomes (data not shown).

\section{Discussion}

In a Canadian population of adults who were at risk for diabetes, we found that higher total NEFA concentrations independently predicted lower beta cell function over 6 years. While we found negative associations with palmitic acid (16:0), oleic acid (18:1 n-9) and linoleic acid (18:2 n-6) for the concentration $(\mathrm{nmol} / \mathrm{ml})$ modelling, no associations were seen for these fatty acids as a proportion, an observation that was further confirmed by multivariate cluster techniques, which found no ability of these individual NEFA to predict beta cell function. We did find that one fatty acid as a mol\%, EPA (20:5n-3), had a positive association with insulin sensitivity. In general, these observations suggest that the absolute size of the total NEFA fraction, rather than its specific composition (aside from EPA), likely influences the pathogenesis of diabetes, at least within a population at risk for diabetes.

The role of total NEFA in the aetiology of diabetes is well documented. Epidemiological studies have shown that higher NEFA associates with lower insulin secretion and a higher risk for developing diabetes [3, 4, 14, 37]. In a cross-sectional analysis of the Reading, Imperial, Surrey, Cambridge, and Kings (RISCK) cohort, total NEFA had a negative association with insulin sensitivity and a particularly strong negative association with beta cell function [13]. Another cross-sectional study found a null association of total NEFA with beta cell function [38], however, C-peptide was used to estimate beta cell function in this study. Experimentally, several potential mechanisms have been elucidated for the role of NEFA on beta cell function, particularly for palmitic acid. Prolonged exposure to elevated NEFA can induce apoptosis in the beta cells, possibly through endoplasmic reticulum stress, formation of ceramides and generation of nitric oxide, as well as impairment of proinsulin production and mitochondrial function $[2,6,39,40]$. One longitudinal study also found an inverse association between total NEFA and beta cell function over 10 years in 77 Japanese individuals with type 2 diabetes [41]; in this study C-peptide was used to estimate beta cell function. The present analysis is the first study to our knowledge to examine the longitudinal association of a broad spectrum of individual NEFA on beta cell function in a large cohort. We found that there was a strong signal of higher total NEFA, palmitic acid, oleic acid and linoleic acid modelled as concentrations with lower beta cell function. No fatty acid as a mol\% had associations with beta cell function. Taken together, these results suggest that it is the absolute size of the circulating NEFA fraction, irrespective of any specific composition of fatty acids, that is responsible for the hypothesised lipotoxic effects of NEFA on the beta cells.

Biologically, in free-living populations, chronic elevation of NEFA may be mediating its association with metabolic outcomes through TAG. In normal metabolism, NEFA enters the liver and assists in the production of TAG that is to be processed into very-low density lipoproteins (VLDL) [42]. As such, higher NEFA may contribute to hypertriglyceridaemia, which is a known risk factor for diabetes. In the sensitivity analysis adjusting for TAG, all associations with beta cell function were attenuated, suggesting that NEFA may in fact be mediating its association with beta cell dysfunction through higher TAG.

There is substantial experimental evidence highlighting the role of increased NEFA and the subsequent increase in insulin resistance via impairment of insulin signalling cascades, as reviewed in previously published articles [43-45]. However, in this longitudinal analysis, we saw in general no association of any individual or total NEFA with hepatic (HOMA2-\%S) or whole-body insulin sensitivity (ISI), except for EPA. There is some evidence [46-48] that EPA may be involved with lowering the risk for diabetes, potentially due to its role in inflammation [49]. However, since we found the association only with HOMA2-\%S and not with ISI, the significant association could be a chance finding, which will require future studies to confirm. We also identified WC as having a powerful attenuating effect on the associations between unadjusted and adjusted models. There are some possible explanations for our null findings for the insulin sensitivity measures and the influence of WC on the results. First, WC may be a strong causal link in previously reported associations between NEFA and 
insulin sensitivity, given the role adipocytes play in metabolism (e.g. leptin and adiponectin). Second, there may be differences in physiology between fasting and postprandial NEFA kinetics that we are not able to investigate but that may explain our null findings for fasting NEFA and insulin sensitivity. For instance, some experimental studies using clamp protocols found that fasting NEFA was a weak predictor of insulin sensitivity compared with postprandial concentrations of NEFA [11, 50]. Inefficiencies in NEFA uptake into the adipose tissue (as seen in individuals with obesity and larger WC) following postprandial TAG lipolysis via lipoprotein lipase may result in NEFA spilling over into the blood and a subsequent increase in circulating NEFA [51], which may be more metabolically active given postprandial activity. Third, the null findings seen may be due to the high-risk population examined in PROMISE, as the majority of participants had a high WC and BMI. It may be that in this population, insulin resistance has become well established and NEFA may not contribute to insulin resistance at this somewhat more advanced stage in the pathogenesis of diabetes.

Few studies have examined the composition of NEFA on metabolic functioning. One recent, well-analysed study used a variety of advanced fatty acid measurement and statistical techniques to explore the multivariate relationship between NEFA composition and components of the metabolic syndrome [52]. Specifically, the authors identified that NEFA 16:1 n-9, 20:1 n-9 and 22:4 n-6 correlated with components of the metabolic syndrome. Another similar study examining diabetes found that 16:0, 18:0, $18: 1,18: 2,18: 3$ may be useful biomarkers to identify healthy individuals compared with those living with diabetes [53]. However, both studies were limited by smaller sample sizes (approximately 100 participants) and the cross-sectional design.

There are a few important limitations to our study. NEFA concentration and composition was only quantified at the baseline visit and as such we cannot investigate whether there were concomitant changes in NEFA and the metabolic measures over time. However, we believe this strengthens our specific objective as the potential impact that reverse causality, due to the tight integration between fatty acid and glucose metabolism, may have on the results is lower. This is also an observational cohort and there may be some residual confounding we have not considered or that could not be measured. Nonetheless, potential covariates were empirically analysed prior to inclusion in the GEE models to best understand and minimise potential confounding. While there may be potential overadjustment due to controlling for WC, both QIC and directed acyclic graph outputs recommended including WC in the GEE model. Nevertheless, we ran sensitivity analyses and identified $\mathrm{WC}$ as the covariate that attenuated the unadjusted results. This attenuation may be due to the fact that higher NEFA may be reflective of greater adipose tissue mass and act as a mediator between the adipose tissue and insulin sensitivity (since adipose tissue is an active endocrine organ).

We used indices of insulin sensitivity and beta cell function estimated from glucose and insulin values collected during OGTTs. While these indices have all been validated against gold standard measures, it is possible our measures may contain some degree of measurement misclassification and are thus not sensitive enough to identify potential associations with NEFA. Finally, our cohort consists of individuals at risk for diabetes, who were primarily female and of European ancestry and while sex and ethnicity did not influence the results presented from this cohort, our findings may not entirely be generalisable to other populations. However, given these limitations, our study also has several strengths, including the longitudinal design and the rigorous statistical techniques and methods applied, which are specifically suited to investigating temporal relationships and to handling the multivariate nature of the data. Finally, our cohort contains highly detailed and comprehensive variable measurements at each collection visit and has both concentration and $\mathrm{mol} \%$ data for the fatty acids.

In conclusion, we found that total NEFA was a strong predictor for lower beta cell function over 6 years, irrespective of the specific composition of the NEFA fraction, suggesting that efforts at lowering circulating total NEFA through medication and/or lifestyle strategies to reduce the risk of diabetes are well warranted. We also found that higher EPA was associated with higher hepatic insulin sensitivity. While future studies are needed to confirm these findings, our results reinforce the importance of continuing to investigate the role of circulating NEFA concentration on the natural history of diabetes.

Acknowledgements The authors thank J Neuman, PV Nostrand, S Kink and A Barnie (all of the Leadership Sinai Centre for Diabetes, Mount Sinai Hospital, Canada) and S Porter and M Marin (both of the Centre for Studies in Family Medicine, University of Western Ontario, Canada) for their expert technical assistance and dedication in their work for PROMISE.

Data availability The datasets generated during and/or analysed during the current study are available from the corresponding author on reasonable request.

Funding This study was supported by grants from the Canadian Diabetes Association (CDA; Grant number: OG-3-14-4574-AH), the Canadian Institutes for Health Research (Grant number: MOP-130458) and the University of Toronto Banting and Best Diabetes Centre. LWJ was supported by a CDA Doctoral Student Research Award; RR was supported by a Heart and Stroke Foundation of Ontario Mid-Career Investigator Award; SBH holds the CDA Chair in National Diabetes Management and the Ian McWhinney Chair of Family Medicine Studies at the University of Western Ontario; RPB holds a Tier II Canada Research Chair in Brain Lipid Metabolism and AJH holds a Tier II Canada Research Chair in Diabetes Epidemiology.

Duality of interest The authors declare that there is no duality of interest associated with this manuscript. 
Contribution statement The authors had the following responsibility: LWJ analysed and interpreted the data and drafted the article; RR and SBH contributed to the conception and design and revised the article intellectual content; ZL acquired the data and revised the article intellectual content; AG assisted with interpretation of the data and revised the article intellectual content; AJH and RPB substantially contributed to the study conception and design, assisted with interpretation of the data and revised the article intellectual content. All authors read and approved the final version. LWJ and AJH have primary responsibility for final content and are the guarantors of this work.

\section{References}

1. Rachek LI (2014) Free fatty acids and skeletal muscle insulin resistance. Prog Mol Biol Transl Sci 121:267-292

2. Cnop M (2008) Fatty acids and glucolipotoxicity in the pathogenesis of Type 2 diabetes. Biochem Soc Trans 36:348-352

3. Paolisso G, Tataranni PA, Foley JE et al (1995) A high concentration of fasting plasma non-esterified fatty acids is a risk factor for the development of NIDDM. Diabetologia 38:1213-1217

4. Salgin B, Ong KK, Thankamony A et al (2012) Higher fasting plasma free fatty acid levels are associated with lower insulin secretion in children and adults and a higher incidence of type 2 diabetes. J Clin Endocrinol Metab 97:3302-3309

5. Kato T, Shimano H, Yamamoto T et al (2008) Palmitate impairs and eicosapentaenoate restores insulin secretion through regulation of SREBP-1c in pancreatic islets. Diabetes 57:2382-2392

6. Maris M, Robert S, Waelkens E et al (2013) Role of the saturated nonesterified fatty acid palmitate in beta cell dysfunction. $\mathrm{J}$ Proteome Res 12:347-362

7. Deguil J, Pineau L, Rowland Snyder EC et al (2011) Modulation of lipid-induced ER stress by fatty acid shape. Traffic 12:349-362

8. Szendroedi J, Frossard M, Klein N et al (2012) Lipid-induced insulin resistance is not mediated by impaired transcapillary transport of insulin and glucose in humans. Diabetes 61:3176-3180

9. Daniele G, Eldor R, Merovci A et al (2014) Chronic reduction of plasma free fatty acid improves mitochondrial function and wholebody insulin sensitivity in obese and type 2 diabetic individuals. Diabetes 63:2812-2820

10. Liang H, Tantiwong P, Sriwijitkamol A et al (2013) Effect of a sustained reduction in plasma free fatty acid concentration on insulin signalling and inflammation in skeletal muscle from human subjects. J Physiol 591:2897-2909

11. Kehlenbrink S, Koppaka S, Martin M et al (2012) Elevated NEFA levels impair glucose effectiveness by increasing net hepatic glycogenolysis. Diabetologia 55:3021-3028

12. Miller MR, Pereira RI, Langefeld CD et al (2012) Levels of free fatty acids (FFA) are associated with insulin resistance but do not explain the relationship between adiposity and insulin resistance in Hispanic Americans: The IRAS Family Study. J Clin Endocrinol Metab 97:3285-3291

13. Johns I, Goff L, Bluck LJ et al (2014) Plasma free fatty acids do not provide the link between obesity and insulin resistance or beta-cell dysfunction: Results of the Reading, Imperial, Surrey, Cambridge, Kings (RISCK) study. Diabet Med 31:1310-1315

14. Charles MA, Eschwège E, Thibult $\mathrm{N}$ et al (1997) The role of nonesterified fatty acids in the deterioration of glucose tolerance in Caucasian subjects: Results of the Paris prospective study. Diabetologia 40:1101-1106

15. WHO, IDF (2006) Definition and diagnosis of diabetes mellitus and intermediate hyperglycaemia: Report of a WHO/IDF consultation. Available from: http://whqlibdoc.who.int/publications/2006/ 9241594934_eng.pdf
16. Matthan NR, Ip B, Resteghini N et al (2010) Long-term fatty acid stability in human serum cholesteryl ester, triglyceride, and phospholipid fractions. J Lipid Res 51:2826-2832

17. Folch J, Lees M, Sloane Stanley GH (1957) A simple method for the isolation and purification of total lipides from animal tissues. $\mathrm{J}$ Biol Chem 226:497-509

18. Nishi S, Kendall CWC, Gascoyne A-M et al (2014) Effect of almond consumption on the serum fatty acid profile: a dose-response study. Br J Nutr 112:1137-1146

19. Johnston LW, Harris SB, Retnakaran R et al (2016) Longitudinal associations of phospholipid and cholesteryl ester fatty acids with disorders underlying diabetes. J Clin Endocrinol Metab 101:25362544

20. Levy JC, Matthews DR, Hermans MP (1998) Correct homeostasis model assessment (HOMA) evaluation uses the computer program. Diabetes Care 21:2191-2192

21. Matsuda M, DeFronzo R (1999) Insulin sensitivity indices obtained from oral glucose tolerance testing: Comparison with the euglycemic insulin clamp. Diabetes Care 22:1462-1470

22. Abdul-Ghani M, Matsuda M, Balas B, DeFronzo R (2007) Muscle and liver insulin resistance indexes derived from the oral glucose tolerance test. Diabetes Care 30:89-94

23. Wareham N, Phillips D, Byrne C, Hales C (1995) The 30 minute insulin incremental response in an oral glucose tolerance test as a measure of insulin secretion. Diabet Med 12:931

24. Retnakaran R, Qi Y, Goran M, Hamilton J (2009) Evaluation of proposed oral disposition index measures in relation to the actual disposition index. Diabet Med 26:1198-1203

25. Hermans MP, Levy JC, Morris RJ, Turner RC (1999) Comparison of insulin sensitivity tests across a range of glucose tolerance from normal to diabetes. Diabetologia 42:678-687

26. Kriska A, Knowler W, LaPorte R et al (1990) Development of questionnaire to examine relationship of physical activity and diabetes in Pima Indians. Diabetes Care 13:401-411

27. Zeger SL, Liang KY (1986) Longitudinal data analysis for discrete and continuous outcomes. Biometrics 42:121-130

28. Greenland S, Pearl J, Robins JM (1999) Causal diagrams for epidemiologic research. Epidemiology 10:37-48

29. Textor J, Hardt J, Knüppel S (2011) DAGitty: A graphical tool for analyzing causal diagrams. Epidemiology 22:745

30. Shrier I, Platt RW (2008) Reducing bias through directed acyclic graphs. BMC Med Res Methodol 8:70

31. Pan W (2001) Akaike's information criterion in generalized estimating equations. Biometrics 57:120-125

32. Barrows BR, Parks EJ (2006) Contributions of different fatty acid sources to very low-density lipoprotein-triacylglycerol in the fasted and fed states. J Clin Endocrinol Metab 91:1446-1452

33. R Core Team (2015) R: A Language and Environment for Statistical Computing. R Foundation for Statistical Computing, Vienna

34. Højsgaard S, Halekoh U, Yan J (2006) The R package geepack for generalized estimating equations. J Stat Softw 15(2):1-11

35. Benjamini Y, Hochberg Y (1995) Controlling the false discovery rate: A practical and powerful approach to multiple testing. J R Stat Soc Ser B Stat Methodol 57:289-300

36. Vandenbroucke JP, von Elm E, Altman DG et al (2007) Strengthening the Reporting of Observational Studies in Epidemiology (STROBE): Explanation and elaboration. PLoS Med 4:e297

37. Pankow JS, Duncan BB, Schmidt MI et al (2004) Fasting plasma free fatty acids and risk of type 2 diabetes: The atherosclerosis risk in communities study. Diabetes Care 27:77-82

38. Rebelos E, Seghieri M, Natali A et al (2015) Influence of endogenous NEFA on beta cell function in humans. Diabetologia 58:2344 2351 
39. Giacca A, Xiao C, Oprescu AI et al (2011) Lipid-induced pancreatic beta-cell dysfunction: Focus on in vivo studies. Am J Physiol Endocrinol Metab 300:E255-E262

40. Xiao C, Giacca A, Lewis GF (2009) The effect of high-dose sodium salicylate on chronically elevated plasma nonesterified fatty acidinduced insulin resistance and beta-cell dysfunction in overweight and obese nondiabetic men. Am J Physiol Endocrinol Metab 297: E1205-E1211

41. Morita S, Shimajiri Y, Sakagashira S et al (2012) Effect of exposure to non-esterified fatty acid on progressive deterioration of insulin secretion in patients with type 2 diabetes: a long-term follow-up study. Diabet Med 29:980-985

42. Nielsen S, Karpe F (2012) Determinants of VLDL-triglycerides production. Curr Opin Lipidol 23:321-326

43. Martins AR, Nachbar RT, Gorjao R et al (2012) Mechanisms underlying skeletal muscle insulin resistance induced by fatty acids: Importance of the mitochondrial function. Lipids Health Dis 11:30

44. Ebbert JO, Jensen MD (2013) Fat depots, free fatty acids, and dyslipidemia. Nutrients 5:498-508

45. Capurso C, Capurso A (2012) From excess adiposity to insulin resistance: The role of free fatty acids. Vasc Pharmacol 57:91-97

46. Steffen BT, Steffen LM, Zhou X et al (2015) n-3 Fatty acids attenuate the risk of diabetes associated with elevated serum nonesterified fatty acids: The multi-ethnic study of atherosclerosis. Diabetes Care 38:575-580
47. Virtanen JK, Mursu J, Voutilainen S et al (2014) Serum omega-3 polyunsaturated fatty acids and risk of incident type 2 diabetes in men: The kuopio ischemic heart disease risk factor study. Diabetes Care 37:189-196

48. Sarbolouki S, Javanbakht MH, Derakhshanian H et al (2013) Eicosapentaenoic acid improves insulin sensitivity and blood sugar in overweight type 2 diabetes mellitus patients: A double-blind randomised clinical trial. Singap Med J 54:387-390

49. Calder PC (2009) Polyunsaturated fatty acids and inflammatory processes: New twists in an old tale. Biochimie 91:791-795

50. Magkos F, Fabbrini E, Conte C et al (2012) Relationship between adipose tissue lipolytic activity and skeletal muscle insulin resistance in nondiabetic women. J Clin Endocrinol Metab 97:E1219E1223

51. Almandoz JP, Singh E, Howell LA et al (2013) Spillover of fatty acids during dietary fat storage in type 2 diabetes: relationship to body fat depots and effects of weight loss. Diabetes 62:1897-1903

52. Dai L, Gonçalves CMV, Lin Z et al (2015) Exploring metabolic syndrome serum free fatty acid profiles based on GC-SIM-MS combined with random forests and canonical correlation analysis. Talanta 135:108-114

53. Liu L, Li Y, Guan C et al (2010) Free fatty acid metabolic profile and biomarkers of isolated post-challenge diabetes and type 2 diabetes mellitus based on GC-MS and multivariate statistical analysis. J Chromatogr B Anal Technol Biomed Life Sci 878:2817-2825 